\title{
INVESTIGATION OF THE SOLID-LIQUID PHASE TRANSITION FOR SnIn ALLOYS BY THE POSITRON ANNIHILATION METHOD
}

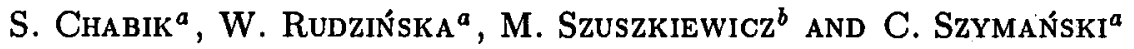 \\ a Institute of Physics, Pedagogical University, Oleska 48, 45-052 Opole, Poland \\ ${ }^{b}$ Institute of Experimental Physics, University of Wroclaw \\ pl. Maxa Borna 9, 50-204 Wrocław, Poland
}

\begin{abstract}
The monovacancy formation energy in Sn-17 wt.\% In and Sn-32 wt.\% In was determined on the basis of the temperature dependence of the peak counting rate for these alloys. A sudden increase in the counting rate was observed between solidus and liquidus temperature. This phenomenon is connected with the formation of large defects acting as positron traps.
\end{abstract}

PACS numbers: $64.70 . \mathrm{Dv}, 61.72 . \mathrm{Cc}, 78.70 . \mathrm{Bj}$

\section{Introduction}

As it is proved by positron annihilation (PA) investigations the behavior of pure tin and indium during the heating to the melting point $\left(T_{M}\right)$ and during the melting is different. The peak counting rate, $F(T)$, for solid indium changes by about 8 percent between room temperature and $T_{M}$, but at $T_{M}$ the $F(T)$ parameter increases by no more than $1-2$ percent $[1,2]$. Positron lifetime for In at $T_{\mathrm{M}}$ decreases by about 2 percent [3]. On the contrary, for pure tin the changes of the anihilation parameters are rather insignificant in the solid state whereas at $T_{\mathrm{M}}$ sudden increase of both $F(T)$ [2] and Doppler broadening $S$ parameter [4] is observed. Thus, it may be concluded that in solid tin the positrons are weakly trapped by the monovacancies, while indium-monovacancies trap positrons strongly.

Monovacancy formation energy, $H_{V_{1}}^{F}$, for pure tin and indium was determined with the use of PA method by many authors. The values of $H_{V_{2}}^{F}$, obtained by different PA techniques for indium, collected by Weiler et al. [5], are between 0.43 and $0.59 \mathrm{eV}$. For tin $H_{V_{1}}^{F}$ was determined mainly from the so-called threshold temperature $T_{\mathrm{T}}$ and is equal to about $0.5 \mathrm{eV}[4,6,7]$. The phase diagram of $\mathrm{Sn}$-In alloy is simple, but the structure and the melting process of $\mathrm{Sn}$-rich samples are rather complicated [8]. The coexistence of amorphous and crystalline phases was observed in sub-microcrystalline solid samples. Thus it may be interesting to know how the various mechanisms of positron trapping for pure metals are reflected in 
the chosen Sn-In alloys: Sn-17 wt.\% In and Sn-32 wt.\% In. The first one has one phase $(\gamma)$ in the solid state, and the second one exhibits the two-phase $(\beta+\gamma)$ structure in the solid state.

\section{Experiment}

The samples were prepared by melting the granulate of the $4 \mathrm{~N}$ pure tin and indium in the rectangular measuring vessel. The heater was placed on the one side of the vessel while at the opposite side the titanium foil ( $8 \mathrm{~mm}$ thick) window was mounted. In this way positrons from ${ }^{22} \mathrm{Na}$ source penetrated to the inside of the sample through the window. After melting the sample was stored for 5 hours at $400^{\circ} \mathrm{C}$. Next, during slow cooling, step by step, the peak counting rate, $F(T)$, was measured. At each temperature the number of coincidence accumulated, during the time of 2 hours, was of the order of $5 \times 10^{4}$. After that, the angular correlation curves (AC) were measured at the room temperature (RT), near the solidus temperature $\left(T_{\mathrm{S}}\right)$ in the solid phase and above the liquidus temperature $\left(T_{\mathrm{L}}\right)$. They were $135^{\circ} \mathrm{C}$ and $215^{\circ} \mathrm{C}$ for $\mathrm{Sn}-17 \mathrm{wt} . \%$ In and $112^{\circ} \mathrm{C}$ and $185^{\circ} \mathrm{C}$ for $\mathrm{Sn}-32 \mathrm{wt} . \% \mathrm{In}$, respectively. All measurements were performed in the protective argon atmosphere under the atmospheric pressure. Temperature was controlled by $\mathrm{NiCr}-\mathrm{Ni}$ thermocouple with an accuracy of $\pm 1^{\circ} \mathrm{C}$. The details of the apparaturs are described in [9]. Positron lifetime spectrum was measured at the RT for a well-annealed Sn-17 wt.\% In sample with the use of a conventional fast-slow spectrometer with resolution function (FWHM) $310 \pm 5$ ps.

\section{Results and discussion}

Figures $1 \mathrm{~b}$ and $2 \mathrm{~b}$ show the results of the measurements of the temperature dependence of the peak counting rate for $\mathrm{Sn}-17 \mathrm{wt} . \%$ In and $\mathrm{Sn}-32 \mathrm{wt} . \%$ In samples, respectively. For clarity, the same results are figured in Figs. 1a and $2 \mathrm{a}$, but they are in the greater scale on the $T$-axis between RT and $250^{\circ} \mathrm{C}$. Solidus and liquidus temperatures marked in the figures are taken from a phase diagram of Sn-In alloy, presented in Ref. [8]. As we can see, in the range from RT to $T_{\mathrm{S}}$, the value of $F(T)$ increases insignificantly. It was assumed that this results from positron trapping at monovacancies. Simple trapping model [10] was used to obtain monovacancy formation energy from the formula

$$
F(T)=\frac{F_{0}+A F_{v} \exp \left(-\frac{H_{V_{1}}^{F}}{k T}\right)}{1+A \exp \left(\frac{H_{V_{1}}^{F}}{k T}\right)},
$$

where $F_{0}$ is the a verage value of $F(T)$ near $\mathrm{RT}, F_{v}$ is the average value of $F(T)$ near $T_{\mathrm{S}}, k$ - Boltzmann constant, $A=\mu \tau_{f} \exp \left(S_{V_{1}}^{F} / k\right)$ with $\tau_{f}$ - positron lifetime in the bulk, $S_{V_{1}}^{F}$ - monovacancy formation entropy, $\mu$ - positron trapping rate per unit vacancy concentration.

Best fitting curves (solid lines in Figs. 1a and 2a) were obtained for $H_{V_{1}}^{F}=$ $0.52 \pm 0.06 \mathrm{eV}$ and $A=(1.4 \pm 0.6) \times 10^{7}$ for $\mathrm{Sn}-17 \mathrm{wt} . \%$ In. Corresponding values 

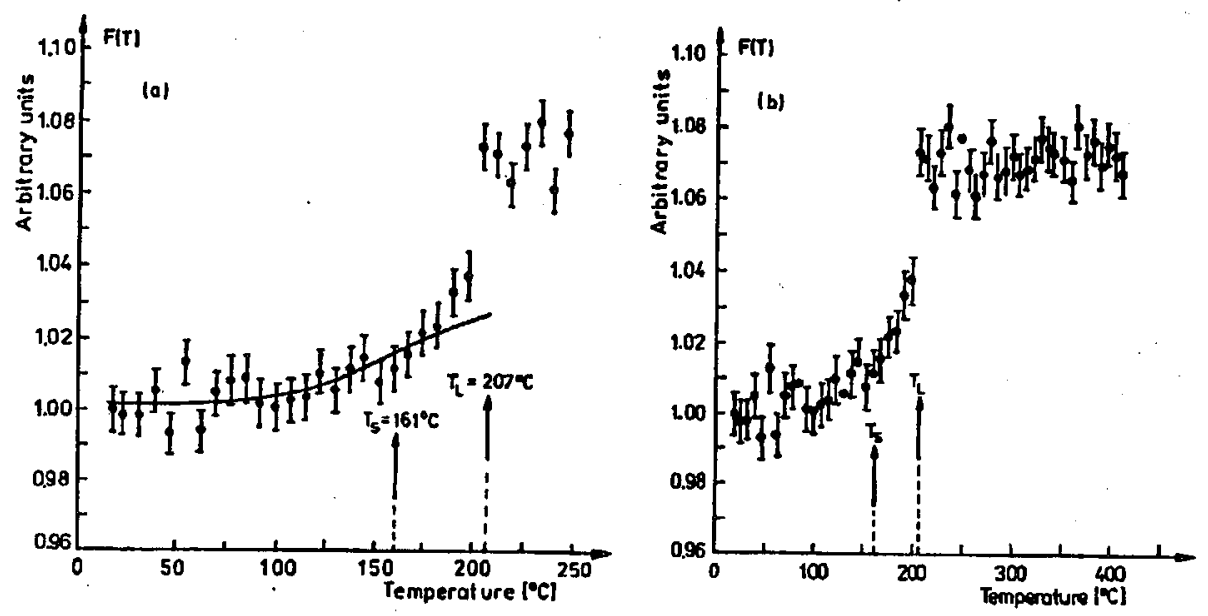

Fig. 1. Temperature dependence of the normalized peak counting rate for Sn-17 wt.\% In alloy in $0-250 \mathrm{~K}(\mathrm{a})$ and $0-400 \mathrm{~K}(\mathrm{~b})$.
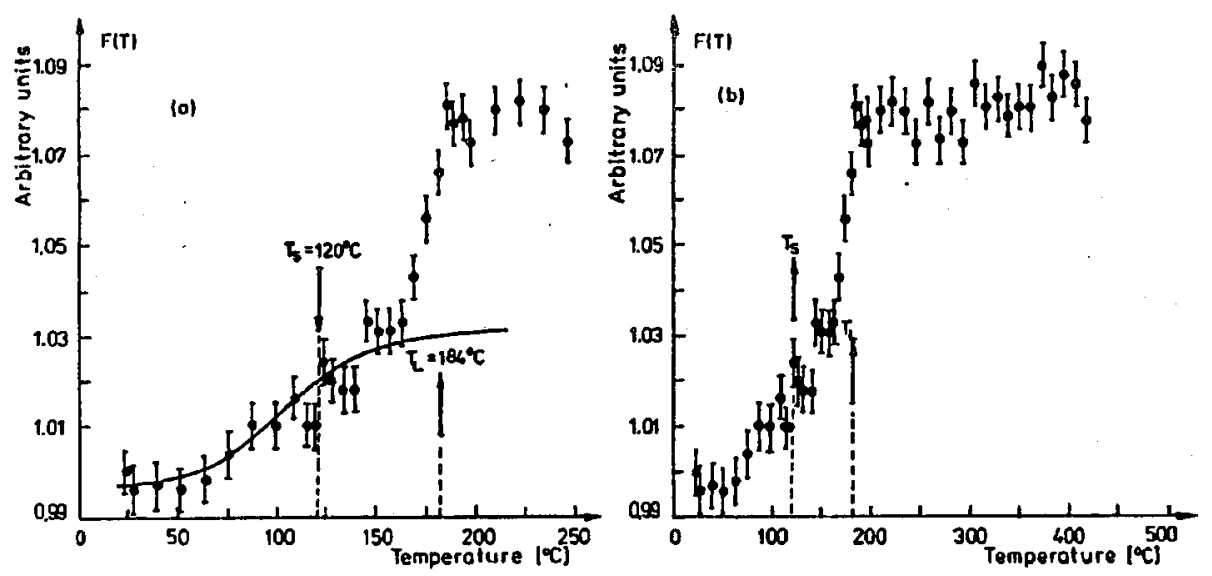

Fig. 2. Temperature dependence of the normalized peak counting rate for $\mathrm{Sn}-32$ wt.\% In alloy in 0-250 K (a) and 0-400 K (b).

for $\mathrm{Sn}-32 \mathrm{wt} . \%$ In are $0.54 \pm 0.04 \mathrm{eV}$ and $(1.7 \pm 0.5) \times 10^{7}$, respectively. The values of $H_{V_{1}}^{F}$ for investigated alloys are comparable with monovacancy formation energy for both pure tin and indium. The obtained values of $A$ are larger than for most metals [11]. Nevertheless, they are of the same order as for pure In $[5,11,12]$. It is an indication that in the investigated alloys positrons are trapped at the In-like monovacancies in the solid phase.

From the RT positron lifetime spectrum for the $\mathrm{Sn}-17 \mathrm{wt} . \%$ In alloy, after the extraction of contributions of positron source and kapton foil, one component was obtained with $\tau=187 \pm 1 \mathrm{ps}$. This value is nearer to the bulk lifetime in pure 
In (192 ps [5]) than in pure Sn (202 ps [13]). Angular correlation curves for pure In and Sn and for the investigated alloys were normalized to the same area. Next, the $R$ parameters were calculated in the similar way described by Trriftshäuser [1]. AC curve measured at $R T$ was taken as a reference. The values of the $R$ parameter were calculated taking into account the area under the peak-part of $\mathrm{AC}$ (from 0 to $2 \mathrm{mrad}$ ) and under the wing-part of $\mathrm{AC}$ (from 8 to $12 \mathrm{mrad}$ ). For pure metals in calculating the $R$ parameter, AC curves measured in RT (somewhat below and above $T_{\mathrm{M}}$ ) were used. The $R$ parameter characterizes the size of the positron trap center. For the alloys the corresponding temperatures were RT, near $T_{\mathrm{S}}$ (solid phase) and above $T_{\mathrm{L}}$ (liquid phase), respectively.

The determined values of the $R$ parameters are collected in Table.

TABLE

The values of the $R$ parameter for solid and liquid $\mathrm{Sn}$, In and their alloys.

\begin{tabular}{c|c|c|c|c}
\hline \hline Phase & Sn & Sn-17 wt.\% In & Sn-32 wt.\% In & In \\
\hline Solid & $0.3 \pm 0.1$ & $0.5 \pm 0.1$ & $0.6 \pm 0.1$ & $1.7 \pm 0.1$ \\
Liquid & $2.4 \pm 0.1$ & $1.8 \pm 0.1$ & $1.9 \pm 0.1$ & $2.0 \pm 0.1$
\end{tabular}

As we can see from Table the size of the trapping center in liquid phase of $\mathrm{Sn}$ and its alloys is larger than that in the solid phase. The nature of these centers is unknown. Nevertheless, it is worth mentioning that during the melting process the behavior of pure $\mathrm{Sn}$ and investigated alloys is similar to that of $\mathrm{Ga}$. For $\mathrm{Ga}$ positron lifetime increases from 190 to $260 \mathrm{ps}$ [14] and $F(T)$ parameter by about 10 percent [9] during the melting. Recently Gramsch at al. [15] have reported a drastic drop in the positron diffusion length for Ga from $1200 \pm 100 \AA$ (solid) to $60 \pm 5 \AA$ after melting. All these facts together with the tendency of $\mathrm{Sn}$ and $\mathrm{Ga}$ to overcooling may indicate that in these liquid metals positrons are trapped at the similar centers as in solid metallic glasses.

\section{Conclusions}

From PA measurements for Sn-17 wt.\% In and Sn-32 wt.\% In alloys the following picture of positron trapping may be deduced. In the liquid phase positrons are trapped in large defects (clusters). During the solidification, when solid Sn-rich crystals grow, those centers rapidly vanish. Near the solidus temperature, when the volume of crystal containing In increases, and In-content increases in them as well, positrons are trapped mainly by monovacancies, which trap positrons also below the solidus temperature. Monovacancy formation energy equals $0.52 \pm 0.006 \mathrm{eV}$ and $0.54 \pm 0.04 \mathrm{eV}$ for $\mathrm{Sn}-17 \mathrm{wt} . \%$ In and Sn-32 wt.\% In, respectively.

Authors are very grateful to Dr. K. Płotkowski who carried out the positron lifetime measurements. 


\section{References}

[1] W. Triffshäuser, Phys. Rev. B 12, 4634 (1975).

[2] W. Rudzińska, S. Chabik, C. Szymaniski, Acta Univ. Wratislav. Mat. Fiz. Astron. 59, 137 (1991).

[3] W. Eckert, J. Bringman, H.E. Schaefer, Condens. Matter 1, Suplement A (1989).

[4] N. Shah, A.L. Catz, Phys. Rev. B 30, 2498 (1984).

[5] W. Weiler, H.E. Schaefer, J. Phys. F, Met. Phys. 15, 1651 (1985).

[6] S. Dedoussis, S. Charalambous, M. Charadalas, Phys. Lett. A 62, 959 (1977).

[7] W. Rudzińska, S. Chabik, C. Szymański, B. Rozenfeld, Cryst. Res. Technol. 22, 11 (1987).

[8] G. Allen, W.A. Jessen, J. Cryst. Growth 70, 546 (1984).

[9] C. Szymański, S. Chabik, J. Pajqk, B. Rozenfeld, Phys. Status Solidi A 60, 375 (1980).

[10] R.N. West, in: Positron in Solids, Ed. P. Hautojärvi, Springer, Berlin 1979, p. 89.

[11] S.M. Kim, W.J.L. Buyers, J. Phys. F, Met. Phys. 8, L103 (1978).

[12] H.E. Schaefer, Phys. Status Solidi A 102, 47 (1987).

[13] P. Mascher, W. Puff, in: Positron Anihilation, Eds. P.C. Jain, R.M. Singru, K.P. Gopinatan, World Sci. Publ., Singapore 1985, p. 527.

[14] W. Brandt, H.F. Waung, Phys. Lett. A 27, 700 (1968).

[15] E. Gramsch, K.G. Lynn, J. Throwe, J. Kanazava, Phys. Rev. Lett. 67, 1282 (1991). 